\title{
A note on comparison results with positive linear operators
}

Meiping Yao ${ }^{*}$ and Aimin Zhao

${ }^{\text {*Correspondence: }}$ yaomp@sxu.edu.cn

School of Mathematical Sciences, Shanxi University, Taiyuan, Shanxi 030006, People's Republic of China

\begin{abstract}
Recently, Jankowski (Appl. Math. Comput. 218:2549-2557, 2011; Appl. Math. Comput. 219:9348-9355, 2013) established four existence results for four difference equations with causal operators. These results are based on four comparison results, respectively. However, the comparison results in the above papers contain inaccuracies. In this paper, we will establish four new comparison results which correct and supplement the comparison results in the above papers. Two examples are given to illustrate our results.
\end{abstract}

MSC: $34 \mathrm{~A} 37 ; 34 \mathrm{~B} 37$

Keywords: positive linear operator; comparison results; difference inequality; boundary condition

\section{Introduction and preliminaries}

The study of differential or difference equations with causal operators has seen a rapid development in the last few years, see the papers [1-5] and the references therein. Recently, existence results for differential or difference equations with causal operators have been obtained by using monotone iterative technique combined with the method of upper and lower solutions; for details, see for example [6-10]. In [7, 8], some existence results for the following boundary value problems of difference equations:

$$
\begin{aligned}
& \left\{\begin{array}{l}
\Delta y(k-1)=(Q y)(k), \quad k \in Z[1, T], \\
g(y(0), y(T))=0,
\end{array}\right. \\
& \left\{\begin{array}{l}
\Delta y(k)=(Q y)(k), \quad k \in Z[0, T], \\
g(y(0), y(T))=0,
\end{array}\right. \\
& \begin{cases}\Delta y(k-1)=Q_{1}(y, z)(k), \quad k \in Z[1, T], \\
\Delta z(k-1)=Q_{2}(z, y)(k), & k \in Z[1, T], \\
g_{1}(y(0), y(T), z(0), z(T))=0, \quad g_{2}(z(0), z(T), y(0), y(T))=0\end{cases}
\end{aligned}
$$

and

$$
\left\{\begin{array}{l}
\Delta y(k)=Q_{1}(y, z)(k), \quad k \in Z[0, T], \\
\Delta z(k)=Q_{2}(z, y)(k), \quad k \in Z[0, T], \\
g_{1}(y(0), y(T), z(0), z(T))=0, \quad g_{2}(z(0), z(T), y(0), y(T))=0,
\end{array}\right.
$$

(c) 2015 Yao and Zhao. This article is distributed under the terms of the Creative Commons Attribution 4.0 International License (http://creativecommons.org/licenses/by/4.0/), which permits unrestricted use, distribution, and reproduction in any medium, provided you give appropriate credit to the original author(s) and the source, provide a link to the Creative Commons license, and indicate if changes were made. 
were obtained based on four comparison results with positive linear operators, respectively. In (1.1)-(1.4), $\Delta y(k)=y(k+1)-y(k), Q, Q_{1}$, and $Q_{2}$ are causal operators (which will be defined in the following), $g \in C\left(R^{2}, R\right), g_{1}, g_{2} \in C\left(R^{4}, R\right)$. However, these comparison results contain inaccuracies and only hold if the linear operators are causal and strictly positive. In this paper, we will give four new comparison results, which hold if the positive linear operators are causal or non-causal. Our results correct the inaccuracies and supplement the comparison results in $[7,8]$. Two examples will be given to illustrate our results.

Let $N_{0}$ be the set of integer numbers and $Z[a, b]=\{a, a+1, \ldots, b\}$ with $a, b \in N_{0}$ and $a<b$. Denote $E=C(Z[a, b], R), E_{0}=C(Z[1, T], R), E_{1}=C(Z[0, T-1], R)$, and $R_{+}=[0,+\infty)$. An operator $Q \in C(E, E)$ is called a causal operator, or nonanticipative, if the following property holds: for each couple of elements of $E$ such that $u(s)=v(s)$, for $a \leq s \leq k, s, k \in$ $Z[a, b]$, as a result $(Q u)(s)=(Q v)(s)$ for $a \leq s \leq k$ with $k<b$ arbitrary. A linear operator $\mathcal{L} \in C(E, E)$ is called a positive linear operator, if $(\mathcal{L} m)(k) \geq 0$ provided that $m(k) \geq 0$, $k \in Z[a, b]$. Similar to [11], a linear operator $\mathcal{L} \in C(E, E)$ is called a strictly positive operator, if $(\mathcal{L} m)(k) \geq 0,(\mathcal{L} m)(k) \not \equiv 0$ provided that $m(k) \geq 0, m(k) \not \equiv 0, k \in Z[a, b]$.

Considering the following inequality with boundary condition:

$$
\left\{\begin{array}{l}
\Delta y(k-1) \leq-M(k) y(k)-(\mathcal{L} y)(k), \quad k \in Z[1, T], \\
y(0) \leq r y(T)
\end{array}\right.
$$

where $M \in C\left(Z[1, T], R_{+}\right), r \geq 0$, and $\mathcal{L} \in C\left(E_{0}, E_{0}\right)$ is a positive linear operator.

Denote $\rho_{1}:=\sum_{i=1}^{T}(\mathcal{L} \mathbf{1})(i) S_{i-1}$ where $\mathbf{1}(k)=1, S_{k}=\prod_{i=1}^{k}[1+M(i)]$ for $k \in Z[1, T]$ and $S_{0}=1$.

We list the following assumptions for convenience.

$\left(\mathrm{H}_{1}\right) \quad \rho_{1} \leq r S_{T}^{-1} \leq 1$ and $S_{T}-r+\rho_{1}>0$,

$\left(\mathrm{H}_{2}\right) \mathcal{L} \in C\left(E_{0}, E_{0}\right)$ is a strictly positive operator and $\rho_{1} \leq r S_{T}^{-1} \leq 1$,

$\left(\mathrm{H}_{3}\right) \mathcal{L} \in C\left(E_{0}, E_{0}\right)$ is a causal operator, $\rho_{1} \leq 1, r S_{T}^{-1} \leq 1$, and $S_{T}-r+\rho_{1}>0$.

Firstly, we state our main comparison results.

Theorem 1.1 Assume that $y \in C(Z[0, T], R)$ and satisfies (1.5). If one of the assumptions $\left(\mathrm{H}_{1}\right)-\left(\mathrm{H}_{3}\right)$ holds, then $y(k) \leq 0, k \in Z[0, T]$.

Remark 1.1 If $\mathcal{L} \in C\left(E_{0}, E_{0}\right)$ is a strictly positive linear operator, then $\rho_{1}>0$. Hence $r S_{T}^{-1} \leq$ 1 implies that $S_{T}-r+\rho_{1}>0$. So one of $\left(\mathrm{H}_{1}\right)-\left(\mathrm{H}_{3}\right)$ ensures $S_{T}-r+\rho_{1}>0$.

Corollary 1.1 Assume that $\mathcal{L} \in C\left(E_{0}, E_{0}\right)$ is strictly positive and causal,

$$
\rho_{1} \leq 1, \quad r S_{T}^{-1} \leq 1 .
$$

If $y \in C(Z[0, T], R)$ and satisfies (1.5), then $y(k) \leq 0, k \in Z[0, T]$.

Remark 1.2 Compared with Lemma 1 in [7], here the operator $\mathcal{L}$ is restricted to be strictly positive and causal. In fact, if $\mathcal{L}$ is only just positive linear, then the condition (1.6) is not sufficient to guarantee that $y(k) \leq 0$ for $k \in Z[0, T]$; see the examples below.

Example 1.1 Consider problem (1.5) with $M(k) \equiv 0,(\mathcal{L} y)(k) \equiv 0$, and $r=1$. Here $\mathcal{L}$ is not strictly positive. Obviously, $\rho_{1}=0<1, r S_{T}^{-1}=1$. Hence the condition (1.6) is fulfilled. However, $y(k) \equiv 1$ satisfies (1.5), which is not non-positive. 
Example 1.2 Consider problem (1.5) with $M(k)=\frac{1}{8}, T=3, r=\frac{1}{5}$, and

$$
(\mathcal{L} y)(k)= \begin{cases}\frac{1}{4} y(2), & k=1, \\ 0, & k=2,3\end{cases}
$$

Here $\mathcal{L}$ is non-causal. It is easy to identify that $0<r S_{T}^{-1}=\frac{8^{3}}{5 \times 9^{3}}<1$ and $\rho_{1}=\sum_{i=1}^{3}(\mathcal{L} \mathbf{1})(i) S_{i-1}=$ $\frac{1}{4}<1$. The condition (1.6) holds. However, $y(0)=-1, y(1)=\frac{2}{9}, y(2)=-5, y(3)=-\frac{40}{9}$ satisfies problem (1.5), but $y(1)$ is not non-positive.

Similar to Theorem 1.1, we can get the following theorem which corrects and complements Lemma 2 in [7].

Theorem 1.2 Assume that $y \in C(Z[0, T], R)$ and satisfies

$$
\left\{\begin{array}{l}
\Delta y(k) \leq-M(k) y(k)-(\mathcal{L} y)(k), \quad k \in Z[0, T-1], \\
y(0) \leq r y(T),
\end{array}\right.
$$

where $M \in C(Z[0, T-1],[0,1)), r \geq 0$, and $\mathcal{L} \in C\left(E_{1}, E_{1}\right)$ is a positive linear operator. If one of the following assumptions holds:

$\left(\mathrm{H}_{1}^{\prime}\right) \quad \tilde{\rho}_{1} \leq r P_{T}^{-1} \leq 1$ and $P_{T}-r+\tilde{\rho}_{1}>0$,

$\left(\mathrm{H}_{2}^{\prime}\right) \mathcal{L} \in C\left(E_{0}, E_{0}\right)$ is a strictly positive linear operator, $\tilde{\rho}_{1} \leq r P_{T}^{-1} \leq 1$,

$\left(\mathrm{H}_{3}^{\prime}\right) \mathcal{L} \in C\left(E_{0}, E_{0}\right)$ is a causal operator, $\tilde{\rho}_{1} \leq 1, r P_{T}^{-1} \leq 1$, and $P_{T}-r+\tilde{\rho}_{1}>0$,

where $\tilde{\rho}_{1}:=\sum_{i=0}^{T-1}(\mathcal{L} \mathbf{1})(i) P_{i+1}, P_{k}=\prod_{i=0}^{k-1}[1-M(i)]^{-1}$ for $k \in Z[1, T]$, then $y(k) \leq 0, k \in$ $Z[0, T]$.

\section{Proofs and applications}

In this section, the proofs of Theorem 1.1 will be given firstly. The proof of Theorem 1.2 is similar and is omitted. Two new comparison results based on Theorems 1.1 and 1.2 will be established secondly.

Proof of Theorem 1.1 It is obvious that $y(k), k=0,1, \ldots, T$, has a maximum value and a minimum value. Let

$$
y\left(k_{*}\right)=\min _{k \in Z[0, T]} y(k), \quad y\left(k^{*}\right)=\max _{k \in Z[0, T]} y(k) .
$$

It is enough to prove that $y\left(k^{*}\right) \leq 0$.

From (1.5) we know that

$$
y(k) \leq \frac{y(k-1)}{1+M(k)}-\frac{(\mathcal{L} y)(k)}{1+M(k)}, \quad k \in Z[1, T] .
$$

Since $\mathcal{L}$ is a positive linear operator,

$$
(\mathcal{L} y)(k)=\mathcal{L}\left(y-y\left(k_{*}\right) \cdot \mathbf{1}\right)(k)+\mathcal{L}\left(y\left(k_{*}\right) \cdot \mathbf{1}\right)(k) \geq y\left(k_{*}\right)(\mathcal{L} \mathbf{1})(k), \quad k \in Z[1, T]
$$

Hence $y(k)$ satisfies the first order linear difference inequality

$$
y(k) \leq \frac{y(k-1)}{1+M(k)}-\frac{y\left(k_{*}\right)(\mathcal{L} \mathbf{1})(k)}{1+M(k)}, \quad k \in Z[1, T] .
$$


By utilizing Theorem 4.4.1 in [12], we have, for any $k_{0} \in Z[0, T-1]$,

$$
y(k) \leq v(k), \quad k \in Z\left[k_{0}, T\right]
$$

where $v(k)$ is the solution of the initial value problem

$$
\left\{\begin{array}{l}
v(k)=\frac{v(k-1)}{1+M(k)}-\frac{y\left(k_{*}\right)(\mathcal{L} \mathbf{1})(k)}{1+M(k)}, \quad k \in Z\left[k_{0}+1, T\right] \\
v\left(k_{0}\right)=y\left(k_{0}\right)
\end{array}\right.
$$

One can easily obtain

$$
v(k)=\frac{y\left(k_{0}\right) S_{k_{0}}}{S_{k}}-y\left(k_{*}\right) \sum_{i=k_{0}+1}^{k} \frac{(\mathcal{L} \mathbf{1})(i) S_{i-1}}{S_{k}}, \quad k \in Z\left[k_{0}, T\right] .
$$

Hence

$$
y(k) \leq \frac{y\left(k_{0}\right) S_{k_{0}}}{S_{k}}-y\left(k_{*}\right) \sum_{i=k_{0}+1}^{k} \frac{(\mathcal{L} \mathbf{1})(i) S_{i-1}}{S_{k}}, \quad k \in Z\left[k_{0}, T\right]
$$

Particularly, using the boundary condition and (2.2), we have

$$
y(T) \leq \frac{y(0)}{S_{T}}-y\left(k_{*}\right) \sum_{i=1}^{T} \frac{(\mathcal{L} \mathbf{1})(i) S_{i-1}}{S_{T}} \leq \frac{r y(T)}{S_{T}}-\frac{\rho_{1} y\left(k_{*}\right)}{S_{T}}
$$

that is,

$$
\left(S_{T}-r\right) y(T)+\rho_{1} y\left(k_{*}\right) \leq 0 .
$$

In view of $r S_{T}^{-1} \leq 1$ and $y(T) \geq y\left(k_{*}\right)$, we have

$$
\left(S_{T}-r+\rho_{1}\right) y\left(k_{*}\right) \leq 0 .
$$

Hence $y\left(k_{*}\right) \leq 0$, since $S_{T}-r+\rho_{1}>0$.

In the following, we will divide the proof into two cases.

Case 1: $k_{*} \leq k^{*}$. From (2.2) we have

$$
y\left(k^{*}\right) \leq \frac{y\left(k_{*}\right) S_{k_{*}}}{S_{k^{*}}}-y\left(k_{*}\right) \sum_{i=k_{*}+1}^{k^{*}} \frac{(\mathcal{L} \mathbf{1})(i) S_{i-1}}{S_{k^{*}}}=\frac{y\left(k_{*}\right)}{S_{k^{*}}}\left[S_{k_{*}}-\sum_{i=k_{*}+1}^{k^{*}}(\mathcal{L} \mathbf{1})(i) S_{i-1}\right] .
$$

Noticing that

$$
S_{k_{*}}-\sum_{i=k_{*}+1}^{k^{*}}(\mathcal{L} \mathbf{1})(i) S_{i-1} \geq 1-\sum_{i=1}^{T}(\mathcal{L} \mathbf{1})(i) S_{i-1}=1-\rho_{1} \geq 0
$$

and the fact that $y\left(k_{*}\right) \leq 0$, we have $y\left(k^{*}\right) \leq 0$. 
Case 2: $0 \leq k^{*}<k_{*} \leq T$. Taking $k_{0}=0$ and $k_{0}=k_{*}$, respectively, in (2.2) we get

$$
y\left(k^{*}\right) \leq \frac{y(0)}{S_{k^{*}}}-y\left(k_{*}\right) \sum_{i=1}^{k^{*}} \frac{(\mathcal{L} \mathbf{1})(i) S_{i-1}}{S_{k^{*}}}
$$

and

$$
y(T) \leq \frac{y\left(k_{*}\right) S_{k_{*}}}{S_{T}}-y\left(k_{*}\right) \sum_{i=k_{*}+1}^{T} \frac{(\mathcal{L} \mathbf{1})(i) S_{i-1}}{S_{T}}
$$

Case 2.1: $\left(\mathrm{H}_{1}\right)$ or $\left(\mathrm{H}_{2}\right)$ holds. Combining with the boundary condition $y(0) \leq r y(T)$, we have

$$
\begin{aligned}
y\left(k^{*}\right) & \leq \frac{r y(T)}{S_{k^{*}}}-y\left(k_{*}\right) \sum_{i=1}^{k^{*}} \frac{(\mathcal{L} \mathbf{1})(i) S_{i-1}}{S_{k^{*}}} \\
& \leq \frac{r y\left(k_{*}\right)}{S_{k^{*}} S_{T}}\left(S_{k_{*}}-\sum_{i=k_{*}+1}^{T}(\mathcal{L} \mathbf{1})(i) S_{i-1}\right)-y\left(k_{*}\right) \sum_{i=1}^{k^{*}} \frac{(\mathcal{L} \mathbf{1})(i) S_{i-1}}{S_{k^{*}}} \\
& =\frac{y\left(k_{*}\right)}{S_{k^{*}}}\left[\frac{r}{S_{T}}\left(S_{k_{*}}-\sum_{i=k_{*}+1}^{T}(\mathcal{L} \mathbf{1})(i) S_{i-1}\right)-\sum_{i=1}^{k^{*}}(\mathcal{L} \mathbf{1})(i) S_{i-1}\right] .
\end{aligned}
$$

Noticing that

$$
\begin{aligned}
& \frac{r}{S_{T}}\left(S_{k_{*}}-\sum_{i=k_{*}+1}^{T}(\mathcal{L} \mathbf{1})(i) S_{i-1}\right)-\sum_{i=1}^{k^{*}}(\mathcal{L} \mathbf{1})(i) S_{i-1} \\
& \quad \geq \frac{r}{S_{T}}-\sum_{i=k_{*}+1}^{T}(\mathcal{L} \mathbf{1})(i) S_{i-1}-\sum_{i=1}^{k^{*}}(\mathcal{L} \mathbf{1})(i) S_{i-1} \\
& \quad \geq \frac{r}{S_{T}}-\sum_{i=1}^{T}(\mathcal{L} \mathbf{1})(i) S_{i-1}=\frac{r}{S_{T}}-\rho_{1} \geq 0
\end{aligned}
$$

we know that $y\left(k^{*}\right) \leq 0$.

Case 2.2: $\left(\mathrm{H}_{3}\right)$ holds. Let $y\left(k_{* *}\right)=\min _{k \in Z\left[0, k^{*}\right]} y(k)$. Then, since $\mathcal{L}$ is causal, we have

$$
y(k) \leq \frac{y(k-1)}{1+M(k)}-\frac{y\left(k_{* *}\right)(\mathcal{L} \mathbf{1})(k)}{1+M(k)}, \quad k \in Z\left[0, k^{*}\right] .
$$

Similar to (2.2), we have

$$
y\left(k^{*}\right) \leq \frac{y\left(k_{* *}\right)}{S_{k^{*}}}-y\left(k_{* *}\right) \sum_{i=k_{* *}}^{k^{*}} \frac{(\mathcal{L} \mathbf{1})(i) S_{i-1}}{S_{k^{*}}}=\frac{y\left(k_{* *}\right)}{S_{k^{*}}}\left[S_{k_{* *}}-\sum_{i=k_{* *}+1}^{k^{*}}(\mathcal{L} \mathbf{1})(i) S_{i-1}\right] .
$$

In view of $y\left(k_{* *}\right) \leq y\left(k^{*}\right)$, we know

$$
\frac{y\left(k_{* *}\right)}{S_{k^{*}}}\left[S_{k^{*}}-S_{k_{* *}}+\sum_{i=k_{* *}+1}^{k^{*}}(\mathcal{L} \mathbf{1})(i) S_{i-1}\right] \leq 0 .
$$


If $S_{k^{*}}-S_{k_{* *}}+\sum_{i=k_{* *+1}}^{k^{*}}(\mathcal{L} \mathbf{1})(i) S_{i-1}>0$, then $y\left(k_{* *}\right) \leq 0$. So

$$
y\left(k^{*}\right) \leq \frac{y\left(k_{* *}\right)}{S_{k^{*}}}\left[S_{k_{* *}}-\sum_{i=k_{* *+}+1}^{k^{*}}(\mathcal{L} \mathbf{1})(i) S_{i-1}\right] \leq \frac{y\left(k_{* *}\right)}{S_{k^{*}}}\left(1-\rho_{1}\right) \leq 0 .
$$

If $S_{k^{*}}-S_{k_{* *}}+\sum_{i=k_{* *+1}}^{k^{*}}(\mathcal{L} \mathbf{1})(i) S_{i-1}=0$, then $y\left(k^{*}\right) \leq \frac{y\left(k_{* *}\right)}{S_{k^{*}}} \cdot S_{k^{*}}=y\left(k_{* *}\right)$. So $y(k)=y\left(k^{*}\right)$ for $k \in Z\left[0, k^{*}\right]$. Thus

$$
y\left(k^{*}\right)=y(0) \leq r y(T) \leq \frac{r y\left(k_{*}\right)}{S_{T}}\left[S_{k_{*}}-\sum_{i=k_{*}+1}^{T}(\mathcal{L} \mathbf{1})(i) S_{i-1}\right] \leq \frac{r y\left(k_{*}\right)}{S_{T}}\left(1-\rho_{1}\right) \leq 0 .
$$

The proof is complete.

In the following we will establish two comparison results which will be used to consider the problems (1.3) or (1.4).

Considering the following inequalities with boundary conditions:

$$
\begin{cases}\Delta p(k-1) \leq-M_{1}(k) p(k)+M_{2}(k) q(k)-\left(\mathcal{L}_{1} p\right)(k)+\left(\mathcal{L}_{2} q\right)(k), & k \in Z[1, T], \\ \Delta q(k-1) \leq-M_{1}(k) q(k)+M_{2}(k) p(k)-\left(\mathcal{L}_{1} q\right)(k)+\left(\mathcal{L}_{2} p\right)(k), & k \in Z[1, T], \\ p(0) \leq r_{1} p(T)+r_{2} q(T), & \\ q(0) \leq r_{1} q(T)+r_{2} p(T), & \end{cases}
$$

where $M_{1}, M_{2}, M_{1}-M_{2} \in C\left(Z[1, T], R_{+}\right), r_{1}, r_{2} \geq 0$, and $\mathcal{L}_{1}, \mathcal{L}_{2}, \mathcal{L}_{1}-\mathcal{L}_{2} \in C\left(E_{0}, E_{0}\right)$ are positive linear operators.

Denote $\rho_{2}=\sum_{i=1}^{T}\left(\left(\mathcal{L}_{1}-\mathcal{L}_{2}\right) \mathbf{1}\right)(i) S_{i-1}^{-}$and $\rho_{3}=\sum_{i=1}^{T}\left(\left(\mathcal{L}_{1}+\mathcal{L}_{2}\right) \mathbf{1}\right)(i) S_{i-1}^{+}$where $S_{k}^{-}=\prod_{i=1}^{k}[1+$ $\left.M_{1}(i)-M_{2}(i)\right], S_{k}^{+}=\prod_{i=1}^{k}\left[1+M_{1}(i)+M_{2}(i)\right]$ for $k \in Z[1, T]$ and $S_{0}^{-}=S_{0}^{+}=1$.

We list the following assumptions for convenience.

$\left(\mathrm{H}_{4}\right) \rho_{3} \leq \frac{r_{1}-r_{2}}{S_{T}^{+}}, \frac{r_{1}+r_{2}}{S_{T}^{-}} \leq 1$, and $S_{T}^{-}-\left(r_{1}+r_{2}\right)+\rho_{2}>0$,

$\left(\mathrm{H}_{5}\right) \mathcal{L}_{1}-\mathcal{L}_{2}$ is a strictly positive linear operator, $\rho_{3} \leq \frac{r_{1}-r_{2}}{S_{T}^{+}}$and $\frac{r_{1}+r_{2}}{S_{T}^{\bar{T}}} \leq 1$,

$\left(\mathrm{H}_{6}\right) \mathcal{L}_{1}, \mathcal{L}_{2}$ are causal operators, $\rho_{3} \leq 1, \frac{r_{1}+r_{2}}{S_{T}^{T}} \leq 1$, and $S_{T}^{-}-\left(r_{1}+r_{2}\right)+\rho_{2}>0$.

Theorem 2.1 Assume that $p, q \in C(Z[0, T], R)$ and satisfy (2.3). If one of the assumptions $\left(\mathrm{H}_{4}\right)-\left(\mathrm{H}_{6}\right)$ holds, then $p(k) \leq 0, q(k) \leq 0, k \in Z[0, T]$.

Proof We only prove the case that condition $\left(\mathrm{H}_{4}\right)$ holds. The others are similar and are omitted. Let $y=p+q$. Then we have

$$
\left\{\begin{array}{l}
\Delta y(k-1) \leq-\left[M_{1}(k)-M_{2}(k)\right] y(k)-\left(\left(\mathcal{L}_{1}-\mathcal{L}_{2}\right) y\right)(k), \quad k \in Z[1, T] \\
y(0) \leq\left(r_{1}+r_{2}\right) y(T)
\end{array}\right.
$$

Condition $\left(\mathrm{H}_{1}\right)$ holds for $M(k)=M_{1}(k)-M_{2}(k), \mathcal{L}=\mathcal{L}_{1}-\mathcal{L}_{2}$, and $r=r_{1}+r_{2}$. By Theorem 1.1 we get $y(k) \leq 0$ for $k \in Z[0, T]$. Thus $p(k)+q(k) \leq 0$ for $k \in Z[0, T]$. Substituting $p(k) \leq$ $-q(k)$ and $q(k) \leq-p(k)$ into (2.3) we have

$$
\left\{\begin{array}{l}
\Delta p(k-1) \leq-\left[M_{1}(k)+M_{2}(k)\right] p(k)-\left(\left(\mathcal{L}_{1}+\mathcal{L}_{2}\right) p\right)(k), \quad k \in Z[1, T] \\
p(0) \leq\left(r_{1}-r_{2}\right) p(T)
\end{array}\right.
$$


and

$$
\left\{\begin{array}{l}
\Delta q(k-1) \leq-\left[M_{1}(k)+M_{2}(k)\right] q(k)-\left(\left(\mathcal{L}_{1}+\mathcal{L}_{2}\right) q\right)(k), \quad k \in Z[1, T] \\
q(0) \leq\left(r_{1}-r_{2}\right) q(T) .
\end{array}\right.
$$

Again condition $\left(\mathrm{H}_{1}\right)$ holds for $M(k)=M_{1}(k)+M_{2}(k), \mathcal{L}=\mathcal{L}_{1}+\mathcal{L}_{2}$, and $r=r_{1}-r_{2}$. By Theorem 1.1, we have $p(k) \leq 0, q(k) \leq 0, k \in Z[0, T]$. The proof is complete.

Considering the following inequalities with boundary conditions:

$$
\begin{cases}\Delta p(k) \leq-M_{1}(k) p(k)+M_{2}(k) q(k)-\left(\mathcal{L}_{1} p\right)(k)+\left(\mathcal{L}_{2} q\right)(k), & k \in Z[0, T-1], \\ \Delta q(k) \leq-M_{1}(k) q(k)+M_{2}(k) p(k)-\left(\mathcal{L}_{1} q\right)(k)+\left(\mathcal{L}_{2} p\right)(k), & k \in Z[0, T-1], \\ p(0) \leq r_{1} p(T)+r_{2} q(T), & \\ q(0) \leq r_{1} q(T)+r_{2} p(T), & \end{cases}
$$

where $M_{1}, M_{2}, M_{1}-M_{2} \in C(Z[0, T-1],[0,1)), r_{1}, r_{2} \geq 0$, and $\mathcal{L}_{1}, \mathcal{L}_{2}, \mathcal{L}_{1}-\mathcal{L}_{2} \in C\left(E_{1}, E_{1}\right)$ are positive linear operators.

Denote $\tilde{\rho}_{2}=\sum_{i=0}^{T-1}\left(\left(\mathcal{L}_{1}-\mathcal{L}_{2}\right) \mathbf{1}\right)(i) P_{i+1}^{-}$and $\tilde{\rho}_{3}=\sum_{i=0}^{T-1}\left(\left(\mathcal{L}_{1}+\mathcal{L}_{2}\right) \mathbf{1}\right)(i) P_{i+1}^{+}$where $P_{k}^{-}=$ $\prod_{i=0}^{k-1}\left[1-M_{1}(i)+M_{2}(i)\right]^{-1}, P_{k}^{+}=\prod_{i=0}^{k-1}\left[1-M_{1}(i)-M_{2}(i)\right]^{-1}$ for $k \in Z[1, T]$.

We list the following assumptions for convenience.

$\left(\mathrm{H}_{4}^{\prime}\right) \quad \tilde{\rho}_{3} \leq \frac{r_{1}-r_{2}}{P_{T}^{+}}, \frac{r_{1}+r_{2}}{P_{T}^{-}} \leq 1$, and $P_{T}^{-}-\left(r_{1}+r_{2}\right)+\tilde{\rho}_{2}>0$,

$\left(\mathrm{H}_{5}^{\prime}\right) \mathcal{L}_{1}-\mathcal{L}_{2} \in C\left(E_{1}, E_{1}\right)$ is a strictly positive linear operator, $\tilde{\rho}_{3} \leq \frac{r_{1}-r_{2}}{P_{T}^{+}}$, and $\frac{r_{1}+r_{2}}{P_{T}^{-}} \leq 1$,

$\left(\mathrm{H}_{6}^{\prime}\right) \mathcal{L}_{1}, \mathcal{L}_{2} \in C\left(E_{1}, E_{1}\right)$ are causal operators, $\tilde{\rho}_{3} \leq 1, \frac{r_{1}+r_{2}}{P_{T}^{-}} \leq 1$, and $P_{T}^{-}-\left(r_{1}+r_{2}\right)+\tilde{\rho}_{2}>0$.

Similar to the proof of Theorem 2.1, a new comparison result based on Theorem 1.2 can be established.

Theorem 2.2 Assume that $p, q \in C(Z[0, T], R)$ and satisfy (2.4). If one of the assumptions $\left(\mathrm{H}_{4}^{\prime}\right)-\left(\mathrm{H}_{6}^{\prime}\right)$ holds, then $p(k) \leq 0, q(k) \leq 0, k \in Z[0, T]$.

Finally, we will address the following fact. In [7, 8], the conditions for causal operators $Q, Q_{1}$, and $Q_{2}$ show that the corresponding positive linear operators $\mathcal{L}, \mathcal{L}_{1}$, and $\mathcal{L}_{2}$ are causal too. Similar to Theorems 3 and 6 of paper [7] and Theorems 3 and 5 of paper [8], existence results of problems (1.1)-(1.4) can be obtained by using the comparison results Theorems 1.1, 1.2, 2.1, and 2.2 in this paper. We will omit the details.

Competing interests

The authors declare that they have no competing interests.

Authors' contributions

All authors contributed equally to the writing of this paper. All authors read and approved the final manuscript.

\section{Acknowledgements}

We are very grateful to the anonymous referees and the associate editor for their careful reading and helpful comments. The work was supported by the Youth Science Foundation of Shanxi Province (Nos. 2014011005-1 and 2014021009-2), the Program for the Top Young Academic Leaders of Higher Learning Institutions of Shanxi (No. 20120304), and Shanxi

Scholarship Council of China (No. 2013-019). 


\section{References}

1. Corduneanu, C: Functional Equations with Causal Operators. Taylor \& Francis, London (2002)

2. Lakshmikantham, V, Leela, S, Drici, Z, McRae, FA: Theory of Causal Differential Equations. Atlantis Studies in Mathmatics for Engineering and Science, vol. 5. World Scientific, Singapore (2010)

3. Corduneanu, C: Existence of solutions for neutral functional differential equations with causal operators. J. Differ. Equ. 168, 93-101 (2000)

4. Drici, Z, McRae, FA, Devi, JV: Differential equations with causal operators in a Banach space. Nonlinear Anal. 62, 301-313 (2005)

5. Agarwal, RP, Zhou, Y, Wang, J, Luo, X: Fractional functional differential equaitons with causal operators in Banach spaces. Math. Comput. Model. 54, 1440-1452 (2005)

6. Ladde, GS, Lakshmikantham, V Vatsala, S: Monotone Iterative Techniques for Nonlinear Differential Equations. Pitman Boston (1985)

7. Jankowski, T: Boundary value problems for difference equations with causal operators. Appl. Math. Comput. 218, 2549-2557 (2005)

8. Jankowski, T: Existence of solutions for a coupled system of difference equations with causal operators. Appl. Math. Comput. 219, 9348-9355 (2013)

9. Jankowski, T: Nonlinear boundary value problems for second order differential equations with causal operators. J. Math. Anal. Appl. 332, 1380-1392 (2007)

10. Geng, FJ: Differential equations involving causal operators with nonlinear periodic boundary conditions. Math. Comput. Model. 48, 859-866 (2007)

11. Niezgoda, M: Shannon like inequalities for $f$-connections of positive linear maps and positive operators. Linear Algebra Appl. 481, 186-201 (2015)

12. Agarwal, RP: Difference Equations and Inequalities: Theory, Methods and Applications, 2nd edn. (2000)

\section{Submit your manuscript to a SpringerOpen ${ }^{\circ}$ journal and benefit from:}

- Convenient online submission

- Rigorous peer review

- Immediate publication on acceptance

- Open access: articles freely available online

- High visibility within the field

- Retaining the copyright to your article 